\title{
LA COMUNICACIÓN DEL CONOCIMIENTO PROPIO EN TESIS: VARIACIÓN ENTRE GRADOS ACADÉMICOS EN LA SECCIÓN DESARROLLO TEÓRICO*
}

\author{
THE COMMUNICATION OF AUTHOR'S OWN KNOWLEDGE \\ WITHIN THESIS: VARIATION AMONG ACADEMIC DEGREES IN \\ THE THEORETICAL FRAMEWORK SECTION
}

\section{PAULINA MEZA}

Universidad de La Serena, La Serena, Chile.

pmeza@userena.cl

\section{BÁRBARA RIVERA}

CIAE, Universidad de Chile, Santiago, Chile.

barbara.rivera@ciae.uchile.cl

\section{RESUMEN}

En la escritura de la Tesis, un estudiante, independientemente de su área y nivel académico, comunica el conocimiento de su disciplina mediante dos fuentes. Por un lado, presenta sus propios aportes, demostrando que es capaz de contribuir en la construcción del conocimiento disciplinar (Fuente Propia); y, por otro, reconoce los aportes de otros autores en su área (Fuente Ajena). En esta investigación, en el marco de un estudio cualitativo, se determinaron los recursos discursivos utilizados en la comunicación del conocimiento propio en la sección Desarrollo Teórico de Tesis de Licenciatura, Magíster y Doctorado en Lingüística. Para ello, se analizó un corpus de 36 Tesis, utilizando categorías de análisis propias (funciones de Fuente Propia) validadas mediante juicio de expertos en distintas etapas. El análisis permitió establecer una serie de diferencias entre las Tesis de los distintos grados, a partir de la frecuencia y la variedad de las funciones analizadas. Entre dichas diferencias destacamos el hecho de que es en el nivel de Doctorado en el que mayor variedad de funciones de la Fuente Propia se utilizan, lo que podría dar cuenta del mayor grado de conocimiento, inserción y consolidación de los tesistas del nivel académico más alto. En conclusión, la investigación se erige como un aporte concreto a la descripción empírica de una sección poco considerada en el estudio del género Tesis, y de una disciplina específica, que es Lingüística. Además, la generación de evidencia granular y altamente especializada

* Esta investigación se inscribe en el proyecto Fondecyt 11010339 "Caracterización de géneros evaluativos como trabajos finales de grado en licenciatura y magíster a través de cuatro disciplinas: Desde los patrones léxico-gramaticales y retórico-estructurales al andamiaje de la escritura académica disciplinar", dirigido por el Dr. René Venegas. 
no solo constituye un avance en el entendimiento de la construcción discursiva del conocimiento disciplinar, sino que también permitirá asistir a quienes enseñan o aprenden sobre la escritura del trabajo final de graduación.

Palabras clave: Comunicación del conocimiento, Tesis, fuente propia, sección Desarrollo Teórico.

\section{ABSTRACT}

Regardless area or academic degree, students communicate discipline knowledge through two sources in their Thesis. On one side, students demonstrate their capability to contribute in the construction of disciplinary knowledge (One's Knowledge). On the other side, students recognize others author's contribution in the discipline (Knowledge of Others). In the context of a qualitative study, we determined the discourse resources used in the communication of One's Knowledge in the Theoretical Framework section of Bachelors, Masters and PhD Thesis within the discipline of Linguistic. We analyzed a corpus of 36 Thesis using our own categories, validated through expert judgment. Results show a series of differences between degrees considering frequency and variety of discourse sources; PhD students use the widest variety of One's Knowledge discourse resources in their Thesis. This phenomenon could be explained by a higher level of knowledge, insertion and consolidation among doctorate students. In conclusion, the present research is a valuable input of empirical evidence for an overlooked section in Thesis genre analysis, and for the discipline of Linguistic. Moreover, the generation of granular level and high specialized evidence furthers the understanding of the discursive construction of disciplinary knowledge, and could assist facilitators and students while writing their Thesis.

Keywords: Knowledge communication, Thesis work, own source, Theoretical Framework.

Recibido: 31.08.2017. Aceptado: 16.04.2018.

\section{INTRODUCCIÓN}

- $\mathrm{n}$ el marco de la escritura académica, una de las actividades de producción ¿de textos que más complejidad reviste es la Tesis, cualquiera sea el grado académico en el que ella se desarrolle (Pereira y Di Stefano, 2007; Tapia y Marinkovich, 2011). Esto, porque su realización implica que el estudiante debe tener las herramientas suficientes no solo para acreditar conocimiento en una determinada área del saber, sino también para construirlo (Badley, 2009) y comunicarlo (Meza, 2013, 2015). Es, quizás, por ello que para un gran número de estudiantes concluir la Tesis implica la inversión de muchos años de trabajo, mientras que para otros resulta una tarea imposible de concluir (Arnoux et al., 2005; Carlino, 2005; Hidalgo y Passarella, 2009). 
Para comunicar el conocimiento es importante conocer los modos de decir propios tanto de la disciplina como del género. Un ejemplo de estos usos particulares es la inclusión de diversas voces o fuentes de información en el texto. Ellos deben ser dominados por quien desea acceder a una comunidad discursiva determinada, lo que no resulta tan sencillo, pues existen escasas instancias formales en que dichos usos se enseñan. Esto, sin duda, obstaculiza la inserción del estudiante a la comunidad a la que se procura pertenecer.

A pesar de la relevancia de las nociones anteriormente descritas, la Tesis, por un lado, sigue siendo un género poco estudiado empíricamente (Venegas, Meza y Martínez, 2013), y la comunicación del conocimiento, por otro, ha sido un aspecto muy poco trabajado en la investigación lingüística en general. De hecho, hasta ahora solo existen trabajos pioneros sobre cómo se comunica el conocimiento en el género Tesis (Meza, 2013, 2015). Además, la gran mayoría de los escasos trabajos que se han preocupado por el estudio empírico de la Tesis, primero, no hacen distinción entre el nivel o grado académico en el que esta se desarrolla (o bien estudian solo un nivel) (Savio, 2010; Tapia y Burdiles, 2012; Venegas, Núñez, Zamora y Santana, 2015); y, segundo, no consideran las características de cada una de las secciones que constituyen el texto en cuestión (Sierra, 2003; Petrić, 2007; Turabian, 2007; Colás, Buendía y Hernández, 2009; Savio, 2010, Tapia y Burdiles, 2012; Meza, 2015, entre otros). Así, abordan el estudio de la Tesis como un género homogéneo en sus distintas secciones y grados académicos. En consecuencia, son casi inexistentes los trabajos que incorporan las variables variación entre grados académicos y apartados de la Tesis; toda vez que se han conjugado estas dos variables, se alude a todos los apartados de la Tesis, por lo que la información presentada es de carácter general (Meza, 2013). Una investigación que se ha ocupado de una sección específica, el Marco Referencial, es la de Tapia y Burdiles (2012), sin embargo, las autoras realizan una descripción en términos de pasos y movidas, y, además, se centran en solo un nivel académico, el de Licenciatura.

En la presente investigación se indaga en un aspecto específico de la comunicación del conocimiento, a saber, las funciones discursivas en la comunicación del conocimiento propio, en la sección Desarrollo Teórico de Tesis de Lingüística de Licenciatura, Magíster y Doctorado. Así, daremos cuenta de las funciones con las que el autor de la Tesis se atribuye el conocimiento a sí mismo (Fuente Propia), en dicha sección, considerando el grado académico al que pertenece. Nos interesa el estudio de esta Fuente en el Desarrollo Teórico, pues tradicionalmente este apartado ha sido definido como la parte de la Tesis en la que el autor se limita a exponer las ideas previas sobre un tema o, dicho de otro modo, lo que otros ya han señalado sobre el tema estudiado (Phillips y Pugh, 2005; Paltridge y Starfield, 2007; Rüger, 2011), y en la que la Fuente predominante es la Ajena (Meza, 2013). Por lo mismo, en las investigaciones existentes no se le ha dado mayor importancia al rol del tesista ni a los aportes que pueda realizar en este apartado en el cual, 
supuestamente, lo esencial son las ideas de otros. Sin embargo, a nuestro juicio, la apropiación que haga el escritor de la Tesis de las ideas de otros puede dar pistas no solo de su grado de conocimiento del tema, sino también de su capacidad crítica frente a ideas ya posicionadas en su ámbito disciplinar.

En este trabajo nos proponemos determinar la variación en la comunicación del conocimiento propio en la sección Desarrollo Teórico de Tesis de Licenciatura, Magíster y Doctorado en Lingüística. Con los resultados obtenidos, entregamos las funciones específicas con las que un autor puede atribuirse la información en el Desarrollo Teórico de su trabajo, considerando el grado académico en el que realiza su Tesis. En consecuencia, el hecho de contar con información empírica y altamente especializada sobre los mecanismos lingüísticos utilizados para la comunicación del conocimiento en la sección en cuestión, por una parte, abre una nueva dimensión de estudio del género Tesis; y, por otra, genera información empírica que puede ser útil en los procesos de alfabetización académica de un género tan complejo como la Tesis. En este sentido, el valor de esta investigación no está en la comparación entre disciplinas, como es habitual en el análisis del discurso académico (Hanania y Akhtar, 1985; Silva, 2012; Yasmin y Mahmood, 2017, entre otros), sino en la descripción acabada de la forma en que el autor se apropia del conocimiento en una disciplina particular y en una sección concreta de la Tesis, a partir de las funciones específicas con las que incorpora su propio conocimiento.

Para llevar a cabo este objetivo, se presenta, a continuación, el marco de referencia que sustenta teóricamente nuestro trabajo y luego el marco metodológico. Posteriormente, se da cuenta de los principales hallazgos de esta investigación, junto a su respectiva discusión. Finalmente, se cierra con las conclusiones del trabajo.

\section{MARCO DE REFERENCIA}

\subsection{Tesis}

La práctica discursiva clave en el paso de la vida estudiantil universitaria a la académico-científica está constituida por la producción de un trabajo de graduación (Moyano, 2000; Bunton, 2002), que permite al escritor-académico novato lograr la entrada a la comunidad discursiva y académica de su interés. Es por esta razón que la Tesis ha sido caracterizada como un rito de iniciación, como aprendizaje progresivo y dominio de los signos de pertenencia de una comunidad (Dardy, Ducard y Maingueneau, 2002; Arnoux, 2006).

Para Moyano (2000) la Tesis es un género discursivo cuya finalidad es informar y acreditar méritos, y su contenido son los resultados de un trabajo de investigación. Por su parte, Koutsantoni (2006) reconoce como característica esencial de 
este género su importante rol en la difusión y ratificación del conocimiento, así como también en la persuasión a la comunidad académica para aceptar a un nuevo integrante junto a los nuevos conocimientos propuestos. Esta autora destaca, además, la importancia de la relación entre los tesistas y los guardianes o porteros del conocimiento (gatekeepers). Esta relación es evidente en dicho género, en el que cobran gran relevancia los procedimientos de atribución del conocimiento a los autores citados, lo que se constituye como base para la construcción y comunicación del nuevo conocimiento disciplinar.

Asimismo, se ha señalado que la Tesis "favorece la apropiación y activación de conocimientos tanto genéricos y estratégicos como específicos del campo en el que se inscribe el proyecto" (Arnoux, 2006:102). Por ello, quien realiza una Tesis debe demostrar suficiencia, tanto en sus conocimientos disciplinares (Aleskerov, 2009) como también en las competencias necesarias para investigar y para divulgar o comunicar sus investigaciones (Thompson, 2005; Colás et al., 2009).

Para Meza (2013), una de las dimensiones que caracteriza la esencia del género Tesis es el diálogo que se produce entre el sujeto que está intentando integrarse a la comunidad discursiva y los autores consagrados de la disciplina. En este diálogo, el autor de la Tesis, por un lado, presenta sus propios aportes, demostrando que es capaz de contribuir en la construcción del conocimiento disciplinar; y, por otro, reconoce los aportes hechos por otros autores en su área, dando la debida atención a su reputación y a sus puntos de vista, atribuyendo a ellos la información. Este fenómeno, que la autora denomina comunicación del conocimiento, "se operativiza mediante la identificación de las formas y funciones que el tesista utiliza tanto para inscribirse a sí mismo en el discurso y para incorporar otras voces" (Meza, 2013: 13-14).

Es precisamente por estas particularidades que las Tesis son instrumentos idóneos para analizar la comunicación del conocimiento, específicamente, la expresión del conocimiento propio y su variación en distintos grados académicos, en una sección específica.

\subsection{La sección Desarrollo Teórico}

En esta investigación nos centramos en la sección dedicada a exponer el sustento teórico de la Tesis. Al respecto, cabe señalar que, dada la gran variedad de etiquetas para aludir a este apartado, tras un análisis estructural de todas las Tesis del corpus, se optó por asignar la denominación Desarrollo Teórico a todas las secciones que cumplan con dicho propósito.

Rüger (2011) recomienda la incorporación de este apartado en la Tesis, a fin de diferenciar claramente los trabajos de otros con la investigación propia. Esta diferenciación cumple dos objetivos, primero, mostrar un panorama general del 
estado de la cuestión, demostrando que existe un vacío en el conocimiento que se necesita cubrir con la Tesis presentada; y, segundo, construir este vacío, que sería la mejor justificación para llevar adelante la Tesis.

Hanania y Akhtar (1985), por su parte, sobre la base del análisis de Tesis de Magíster, dan cuenta de algunas características léxico-gramaticales del apartado en cuestión. Las autoras señalan que en esta sección hay un marcado cambio hacia el tiempo pasado y un ligero aumento en los perfectivos, formas que resultarían apropiadas para la presentación de trabajos que se han realizado antes de la investigación presentada. Un hecho que parece curioso para las autoras es que no existe un cambio de formas activas a pasivas, lo que les da a entender que estas últimas no son necesarias para la revisión de la literatura existente. Este hecho, para ellas, es independiente de la disciplina.

Por otro lado, Paltridge y Starfield (2007) identifican que, para la escritura de la Tesis son necesarias ciertas habilidades de escritura, tales como resumir y criticar investigaciones previas; lo que se lograría a través de la adopción de una postura utilizando verbos de reporte y evaluativos. Esto confirma nuestro planteamiento que, para escribir la Tesis, es necesario conocer y dominar las formas específicas de comunicar el conocimiento en un género determinado y en un apartado específico. Asimismo, estos autores, en concordancia con Rüger (2011), señalan que uno de los objetivos más importantes de la sección analizada es contextualizar la propia investigación. En consecuencia, para Paltridge y Starfield (2007) este apartado debería describir y sintetizar la mayor cantidad posible de estudios relacionados con el tema de la investigación.

Phillips y Pugh (2005) reconocen que el objetivo del Desarrollo Teórico es demostrar que se tiene una comprensión completamente profesional de la teoría previa en una cierta disciplina. Así, de acuerdo con estos autores, para presentarse como un investigador capacitado y realizar una buena revisión de la literatura, es necesario dar cuenta de los desarrollos, las controversias y los avances actuales más interesantes sobre el tema tratado.

Por otra parte, desde un enfoque prescriptivo, algunas instituciones de educación superior han diseñado manuales para orientar a sus estudiantes en la escritura de una Tesis. En este tipo de documentos se afirma que en la sección en cuestión se debe presentar una selección de información relevante para el problema de investigación y puede ir dividido en partes o capítulos, donde se contempla la inclusión de elementos como tablas, gráficos e ilustraciones (USACH, 2008; UDLAP, 2012). Si bien estas afirmaciones son bastante generales y no se sustentan en un trabajo empírico, nos permiten realizar una caracterización desde otra mirada de la sección en cuestión.

Es importante señalar que, si bien la estructura de la Tesis es muy variable (Meza, 2013), la gran mayoría de los autores concuerdan en que el Desarrollo Teórico es una sección obligatoria, independientemente del nivel en el que se realice 
la Tesis, el enfoque metodológico seguido o las distintas denominaciones con que pueda aparecer (Bui, 2013).

\subsection{La comunicación del conocimiento}

Siguiendo a Meza (2013, 2015), entendemos la comunicación del conocimiento como el diálogo que se produce en la práctica discursiva científica para hacer circular el conocimiento. Este diálogo se instancia en los textos mediante diversos recursos lingüísticos y discursivos que se encuentran restringidos por las particularidades del discurso académico.

Según la autora, dicho intercambio comunicativo se realiza en los textos mediante los recursos que el autor utiliza, por una parte, para inscribirse en el discurso y, por otra, para incluir a otros participantes en su discurso a través de los mecanismos de citación. Así, en la comunicación del conocimiento existe una articulación entre la información atribuida al autor de la Tesis (Fuente Propia) y aquella cuyos responsables son los autores citados (Fuente Ajena). A partir de la interacción entre la Fuente Propia y la Ajena, surge un tercer tipo de fuente para comunicar el conocimiento, que es la Interactiva. En esta la información presentada proviene del diálogo o la interacción entre el tesista y los autores que ha citado.

En el estudio de la comunicación del conocimiento, tal como lo propone Meza $(2013,2015)$, se analizan tanto las formas como las funciones con que la información atribuida a cada una de las fuentes se incorpora en el texto. Específicamente, la función, que es la categoría de nuestro interés, se entiende como "categorías pragmáticas que reflejan la intencionalidad del tesista en el texto, vale decir, la intención con que el autor de la Tesis incorpora ciertos fragmentos en su trabajo" (Meza, 2013:140). Ello se sustenta sobre la base de la idea de que hemos desarrollado formas específicas de usar el lenguaje para conseguir nuestros propósitos (Hyland, 2003). De este modo, cada fragmento se incorpora con un motivo concreto y una finalidad que quedará plasmada en el texto.

En la presente investigación nos centraremos solo en la Fuente Propia, esto es, la información atribuida al autor de la Tesis; particularmente, se analiza las funciones con las que esta información es incorporada en la sección Desarrollo Teórico de la Tesis.

\section{MARCO METODOLÓGICO}

\subsection{Tipo de investigación}

La investigación planteada se enmarca en un enfoque metodológico de corte cua- 
litativo, su alcance es exploratorio-descriptivo y su diseño es de tipo no experimental. Las variables son de tipo nominal, y corresponden a: recursos lingüísticodiscursivos utilizados para comunicar el conocimiento propio (funciones, específicamente), grado de especialización académica y sección de la Tesis. Además, es un estudio de naturaleza transeccional, ya que se recolectó Tesis realizadas durante una década (2000 a 2009) en la disciplina y grados académicos propuestos, que serán analizadas sin considerar los efectos del tiempo sobre sus características.

\subsection{Objetivos y preguntas de investigación}

Las preguntas que guían esta investigación son:

a) ¿Con qué funciones se comunica el conocimiento propio en la sección Desarrollo Teórico de las Tesis de Licenciatura, Magíster y Doctorado en Lingüística?

b) ¿Existe variación entre grados académicos en las funciones utilizadas para comunicar el conocimiento propio en la sección Desarrollo Teórico de las Tesis de Licenciatura, Magíster y Doctorado en Lingüística?

Para concretizar estas preguntas se plantea como objetivo general determinar la variación en la comunicación del conocimiento propio en la sección Desarrollo Teórico de las Tesis de Licenciatura, Magíster y Doctorado en Lingüística. Para ello, se identificó las funciones discursivas utilizadas para comunicar el conocimiento propio en la sección Desarrollo Teórico de dichas Tesis; y, luego, se comparó dichas funciones entre grados académicos en la sección mencionada.

\subsection{Descripción del corpus}

El corpus utilizado, denominadoTeLing3N, está conformado por 36 Tesis de los niveles de Licenciatura, Magíster y Doctorado en Lingüística. Para determinar la muestra, que es de tipo no probabilística intencionada (Albert, 2006; Salinas, 2010), se aplicaron los siguientes criterios de inclusión, sustentados en nuestros intereses investigativos: a) Tesis de tres grados académicos, b) Desarrolladas en universidades chilenas, c) Escritas en español, d) Realizadas entre el 2000 y el 2009, e) De Lingüística, f) Escritas por un solo autor. Las Tesis que cumplieron con todos estos criterios se encuentran en dos universidades en Chile: la Pontificia Universidad Católica de Valparaíso (en adelante PUCV) y la Universidad de Concepción (en adelante UDEC). En resumen, las Tesis analizadas del corpus TeLing $3 \mathrm{~N}$ se distribuyen de la siguiente forma: 
Tabla I. Distribución de las Tesis seleccionadas.

\begin{tabular}{|c|c|c|c|}
\hline \multirow{2}{*}{} & \multicolumn{3}{|c|}{ TESIS } \\
\cline { 2 - 4 } & \multicolumn{3}{|c|}{ LINGÜÍSTICA } \\
\cline { 2 - 4 } & PUCV & UDEC & TOTAL \\
\hline LICENCIATURA & 9 & 3 & 12 \\
\hline MAGÍSTER & 3 & 9 & 12 \\
\hline DOCTORADO & 9 & 3 & 12 \\
\hline TOTALES & 21 & 15 & 36 \\
\hline
\end{tabular}

\subsection{Procedimientos}

Los procedimientos llevados a cabo para realizar el análisis empírico los dividimos en etapas y fases, que detallamos a continuación.

1) Conformación del corpus: La fase más importante de esta etapa fue la división del corpus entre uno de prueba y uno de investigación (33 y 67\% de los textos, respectivamente).

2) Análisis estructural de las Tesis del corpus: Esta etapa fue necesaria debido a la gran variedad de secciones, así como de etiquetas para denominarlas. Este análisis permitió obtener apartados comunes a todas las Tesis del corpus y, así, realizar de mejor manera el análisis comparativo de la sección Desarrollo Teórico.

3) Análisis del corpus de prueba: Se utilizó el software ATLAS.ti para el etiquetaje manual del corpus. La parte final de esta etapa contempló una revisión general del análisis del corpus de prueba para corregir posibles errores cometidos durante este primer etiquetaje.

4) Validación de las categorías de análisis del corpus de prueba: Esto fue necesario porque desarrollamos una investigación de carácter exploratorio y de índole inductivo-deductivo.

Esta validación tuvo tres fases: a) Revisión del análisis por parte de una tercera investigadora; b) Triangulación de las categorías de análisis obtenidas y del método utilizado para recogerlas. Se contó con la colaboración de cuatro expertos de las universidades Pompeu Fabra (España) y PUCV (Chile); c) Juicio de evaluadores expertos, en el cual un grupo de investigadores evaluó cada una de las categorías de análisis mediante una escala Likert. El resultado de esta evaluación arrojó un porcentaje de acuerdo de $83,13 \%$, por lo que nuestras categorías superan el valor de referencia mínimo para la validación (Blessing y Chakrabarti, 2009). Asimis- 
mo, el cálculo del coeficiente Kappa de Fleiss indicó que el grado de acuerdo entre jueces es de 0,83, lo que, en términos de Landis y Koch (1977), corresponde a un acuerdo casi perfecto. Finalmente, se ajustaron las categorías según las observaciones incorporadas por los jueces.

5) Análisis y revisión del corpus de investigación: Este análisis fue sometido a los mismos procedimientos de validación mencionados para el corpus de prueba. En esta fase, el grado de acuerdo entre jueces fue de 0,73, lo que corresponde a un acuerdo considerable (Landis y Koch, 1977). Por su parte, el porcentaje de acuerdo alcanzó un 73,26\%, número que supera el valor de referencia mínimo para la validación (Blessing y Chakrabarti, 2009). Finalmente, se realizó la revisión del $100 \%$ del corpus, esto es, la suma del corpus de prueba e investigación.

Tras estas etapas y fases, se obtuvieron las categorías de análisis para el estudio de la comunicación del conocimiento en Tesis de Lingüística. En esta investigación, se presenta los resultados de las 31 funciones asociadas a la Fuente Propia.

\section{ANÁLISIS Y DISCUSIÓN DE RESULTADOS}

En este apartado se da cuenta de cómo se presentan, en la sección Desarrollo Teórico de las Tesis analizadas, las categorías de análisis para la comunicación del conocimiento propio, levantadas a partir del corpus.

En la Tabla II se presenta la proporción de funciones de la Fuente Propia utilizadas en la sección Desarrollo Teórico según su frecuencia de aparición en cada grado académico. Para mostrar estos resultados, se organizó las categorías de análisis indicando el rango que, de acuerdo con su frecuencia relativa, ocupa cada una en cada grado académico. Así, se ha establecido tres rangos, los cuales fueron determinados por el número total de categorías en cada nivel. De este modo, las categorías halladas se separaron en tres grupos, que representan tres tipos de frecuencia, identificados por tres colores: en negro las formas de alta frecuencia, en gris medio las de frecuencia media, en gris claro las de baja frecuencia, y en blanco las categorías con frecuencia cero. Luego, se reorganizó las categorías presentadas en la tabla, ordenándolas alfabéticamente y manteniendo el color que identifica el rango en el cual se encuentra cada una en cada nivel, a fin de observar más claramente las diferencias que existen entre distintos grados académicos. Es importante señalar que este método de organización de los datos ha sido utilizado en otras investigaciones similares validadas por distintos expertos disciplinares (Meza, 2013, 2015). 
En la columna de la izquierda de la Tabla II se indican los nombres de las categorías de análisis y en las columnas siguientes los grados académicos: Licenciatura (L), Magíster (M) y Doctorado (D). Cabe agregar también que en los ejemplos presentados de aquí en adelante se utilizó un código en el que se indican diversos datos de la Tesis desde donde fue extraído cada caso, específicamente, la universidad (PUCV o UDEC), el grado académico (LIC, MAG o DOCT), el año y el número del texto en el corpus. Así, por ejemplo, cada código queda constituido de la siguiente forma: [UDECMAG2007_38].

Tabla II. Distribución funciones Fuente Propia en la sección Desarrollo Teórico.

\begin{tabular}{|c|c|c|c|}
\hline FUNCIONES FUENTE PROPIA DESARROLLO TEÓRICO & $\mathrm{L}$ & M & $\mathrm{D}$ \\
\hline 1. Definir o explicar un concepto o teoría & 0 & 0,53 & 4,6 \\
\hline 2. Caracterizar metodológicamente la propia investigación & 4,19 & 4,81 & 2,45 \\
\hline 3. Describir aspectos metodológicos propuestos & 0,6 & 0,53 & 1,44 \\
\hline 4. Ejemplificar los resultados o hallazgos de la investigación & 1,2 & 0 & 2,01 \\
\hline 5. Emitir juicios sobre algún aspecto de la Tesis & 10,18 & 6,42 & 5,76 \\
\hline 6. Enmarcar la investigación o un concepto relevante para ella & 1,2 & 0,53 & 0 \\
\hline 7. Explicar un artefacto icónico propio & 0,6 & 0,53 & 6,19 \\
\hline 8. Exponer resultados o hallazgos de la investigación & 1,8 & 0 & 0,29 \\
\hline 9. Indicar aportes & 0,6 & 0,53 & 1,29 \\
\hline 10. Indicar dificultades & 0,6 & 1,07 & 2,16 \\
\hline 11. Indicar limitaciones & 0 & 0,53 & 0,58 \\
\hline 12. Indicar notación & 0 & 0 & 0,29 \\
\hline 13. Indicar objetivos & 2,4 & 2,67 & 0,58 \\
\hline 14. Indicar organización & 40,72 & 39,04 & 35,83 \\
\hline 15. Indicar procedimientos & 0 & 3,74 & 3,88 \\
\hline 16. Indicar proyecciones & 0 & 0 & 0,29 \\
\hline 17. Indicar vacíos/ necesidades en el conocimiento & 1,2 & 2,14 & 1,29 \\
\hline 18. Introducir un concepto & 0 & 0 & 0,86 \\
\hline 19. Justificar & 16,77 & 17,65 & 9,64 \\
\hline 20. Plantear hipótesis o supuestos & 1,8 & 3,21 & 1,01 \\
\hline 21. Presentar desarrollo del análisis & 0 & 0 & 3,17 \\
\hline 22. Problematizar & 5,99 & 6,95 & 3,45 \\
\hline 23. Proponer aspectos teóricos & 0,6 & 0 & 2,73 \\
\hline 24. Realizar interpretaciones o deducciones & 4,79 & 4,28 & 2,16 \\
\hline 25. Realizar recomendaciones & 1,8 & 2,67 & 4,75 \\
\hline 26. Sintetizar algún aspecto de la Tesis & 2,99 & 2,14 & 2,59 \\
\hline 27. Superar dificultad & 0 & 0 & 0,72 \\
\hline
\end{tabular}


En primer lugar, cabe señalar que toda vez que, para comunicar el conocimiento en una Tesis, la información se atribuye al autor de la misma, esto es la Fuente Propia, se hace mediante 31 funciones (Meza, 2013, 2015). De ese total, que corresponde a la Tesis en general, en la sección Desarrollo Teórico aparecen solo 27 funciones, lo que constituye el 87,1\%. Dicho de otro modo, en la sección mencionada aparece solo un porcentaje de todas las funciones inicialmente descritas para la Fuente Propia. Más específicamente, en ninguno de los tres niveles encontramos las funciones: Expresar agradecimientos, Corroborar hipótesis de investigación, Delimitar responsabilidades y Refutar hipótesis de la investigación. Esto podría deberse a una cuestión de afinidad función-sección, vale decir, resulta esperable que ciertas funciones aparezcan en secciones específicas, a fin de ayudar a cumplir el propósito comunicativo de los apartados. Así, lo que se espera que aparezca en la sección analizada es la exposición del sustento teórico de la investigación, con el fin de diferenciar claramente los trabajos de otros de la investigación propia y crear, así, un nicho o vacío en el conocimiento (Rüger, 2011). Por el contrario, y de acuerdo con los hallazgos obtenidos, no resulta esperable que en la sección Desarrollo Teórico se presenten, por ejemplo, hipótesis o se expresen agradecimientos, pues ellos están reservados a secciones de la Tesis.

Al realizar el análisis por cada grado académico, se encontró que tanto en Licenciatura como en Magíster se presentan 19 de las 27 funciones, aunque no corresponden exactamente a las mismas; mientras que en Doctorado aparecen 26 de las 27, ausentándose solo Enmarcar la investigación o un concepto relevante para ella. Esta mayor variedad de funciones de la Fuente Propia en Doctorado podría sugerir que, en el nivel académico superior, existe una mayor destreza para comunicar el conocimiento, puesto que estos estudiantes tienen la capacidad de, en una misma sección, comunicar el conocimiento propio a través de una mayor variedad de funciones. Ahora bien, esta mayor destreza podría atribuirse al hecho de que en el nivel académico más alto los estudiantes ya han desarrollado su identidad de autor, lo que, siguiendo a Abasi y Graves (2008), se debería a la práctica adquirida en escritura académica, pues mediante ella los estudiantes "simultaneously construct, and are constructed, by their texts" (Abasi y Graves, 2008:222).

Otro fenómeno que apoya al análisis anterior son las funciones exclusivas de cada grado académico. En nuestro análisis se observa que en el apartado Desarrollo Teórico no existen funciones privativas de las Tesis de Licenciatura ni de las de Magíster. En cambio, en Doctorado sí aparecen y corresponden a: Presentar desarrollo del análisis, Introducir un concepto, Indicar notación, Indicar proyecciones y Superar dificultad. Por tanto, es Doctorado el único grado que posee funciones exclusivas para comunicar el conocimiento propio en la sección Desarrollo Teórico. Esto comprueba la mayor experticia de los estudiantes de Doctorado, no solo por la variedad de funciones que utilizan para dar cuenta de sus aportes, sino también 
porque las funciones utilizadas muestran que son capaces de realizar contribuciones que no aparecen en otros niveles como, por ejemplo, introducir un nuevo concepto en su área. Creemos que esto se debe a que los estudiantes de Doctorado tienen mayor conciencia de que el escritor de Tesis debe demostrar suficiencia en sus conocimientos disciplinares (Aleskerov, 2009) si quiere ser aprobado con éxito por los gatekeepers de su comunidad. En esta misma línea, los tesistas doctorales se esforzarían más por demostrar que han cumplido con uno de los requerimientos centrales de la tesis de Doctorado, esto es, haber hecho al menos un nuevo descubrimiento o una nueva contribución al conocimiento dentro del tema de especialización elegido (Rüger, 2011).

Del mismo modo, existen otras funciones que solo se encuentran en las Tesis de postgrado, a saber: Definir o explicar un concepto o teoría, Indicar limitaciones e Indicar procedimientos. Desde nuestro punto de vista, la presencia de funciones privativas de los niveles de postgrado y de Doctorado, pero no de Licenciatura, responde al hecho de que el tesista de postgrado está más inserto en su comunidad discursiva, lo que podría implicar, por una parte, que ha leído más, por lo que puede aportar más información de manera más diversificada; y, por otra, que, al haber leído más a los autores de su disciplina, conoce mejor la forma de escribir en ella.

Ahora bien, el análisis de la frecuencia de aparición de las funciones estudiadas permite distinguir entre distintos grados académicos. Las funciones que evidencian diferencias entre los tres grados académicos son, primero, Definir o explicar un concepto o teoría, cuya frecuencia va aumentando a medida que se avanza en nivel académico, siendo inexistente en Licenciatura, de frecuencia baja en Magíster $(0,53)$ y alta en Doctorado $(4,6)$. Esto implica que son los estudiantes de Doctorado quienes más definiciones o explicaciones propias incorporan en el sustento teórico de su trabajo, hecho que podría atribuirse a la mayor cantidad de conocimientos que poseen y al mayor grado de originalidad que exige la Tesis de Doctorado (Lovitts y Wert, 2009). Esto mismo explica el hecho de que en Licenciatura sean inexistentes las definiciones o explicaciones propias de los tesistas.

Una segunda función que nos permite diferenciar entre los tres grados académicos es Ejemplificar los resultados o hallazgos de la investigación, la cual es inexistente en Magíster, de frecuencia baja en Licenciatura $(1,2)$ y media en Doctorado $(2,01)$. En consecuencia, son las Tesis de Doctorado donde aparecen mayor cantidad de ejemplos creados por los tesistas, lo que podría deberse a la mayor complejidad de los temas tratados en este nivel. Otra interpretación posible es que en Doctorado hay mayor conocimiento y dominio de la escritura académica, más específicamente, mayor consideración por el lector, lo que deriva en la presentación de más ejemplos para que el lector pueda lograr una mejor comprensión del tema expuesto.

Tercero, la función Exponer resultados o hallazgos de la investigación no se en- 
cuentra en las Tesis de Magíster, es de frecuencia media en Licenciatura $(1,8)$ y baja en Doctorado (0,29). Así, a pesar de que la función central del Desarrollo teórico es presentar los avances que han hecho otros autores sobre el tema (Rüger, 2011), los estudiantes de Licenciatura son quienes, en dicha sección, presentan más cantidad de resultados de su propia investigación. Ello evidencia mayor desconocimiento de los recursos lingüísticos que permiten diferenciar el conocimiento propio y ajeno, así como también de los propósitos comunicativos específicos de cada sección.

Por último, Proponer aspectos teóricos es otra de las funciones que permite diferenciar entre los tres niveles académicos en estudio. Esta función es inexistente en Magíster, de frecuencia baja en Licenciatura $(0,6)$ y media en Doctorado $(2,73)$. Estos resultados muestran que son los tesistas de Doctorado quienes con mayor frecuencia presentan sus propias propuestas teóricas para el tópico tratado, hecho que podría atribuirse a su mayor grado de inserción disciplinar y, en consecuencia, mayor dominio de su área de estudio. Otra interpretación posible dice relación con el hecho de que en Doctorado se exige mayor originalidad (Lovitts y Wert, 2009), por lo que la propuesta de aspectos teóricos resulta más frecuente.

Por otro lado, identificamos una función que permite distinguir entre las Tesis de pre y postgrado. Se trata de Indicar vacíos o necesidades en el conocimiento, la cual tiene una frecuencia baja en las Tesis de pregrado y media en las de postgrado. Si bien podría pensarse que esta es una función propia de la Introducción, otros autores, desde el punto de vista de pasos y movidas, también han dado cuenta de su presencia en el Desarrollo Teórico (Kwan, 2006; Silva, 2012). Además, es importante recordar que esta última sección tiene como objetivo mostrar un panorama general del estado de la cuestión, demostrando la existencia de un nicho o vacío de conocimiento que la investigación llenaría (Rüger, 2011), por lo que no resulta tan extraña la presencia de enunciados que apunten a un vacío investigativo.

Otra distinción que nos permite realizar el análisis es entre las Tesis de Licenciatura y Magíster versus las de Doctorado a través de las frecuencias de nueve funciones. Ellas son: (a) Caracterizar metodológicamente la propia investigación (frecuencia alta en Licenciatura y Magíster, media en Doctorado), (b) Describir aspectos metodológicos propuestos (frecuencia media en Doctorado, baja en Licenciatura y Magíster), (c) Explicar un artefacto icónico propio (baja frecuencia en Licenciatura y Magíster, alta en Doctorado), (d) Indicar dificultades (baja frecuencia en Licenciatura y Magíster, media en Doctorado), (e) Indicar aportes (baja frecuencia en Licenciatura y Magíster, media en Doctorado), (f) Indicar objetivos (frecuencia media en Licenciatura y Magíster, baja en Doctorado), (g) Plantear hipótesis o supuestos (frecuencia media en Licenciatura y Magíster, baja en Doctorado), (h) Realizar interpretaciones o deducciones (alta frecuencia en Licenciatura y Magíster, media en Doctorado), (i) Realizar recomendaciones (frecuencia alta en Doctora- 
do, media en Licenciatura y Magíster).

A continuación, se discutirán aquellas que resultan más interesantes. En primer lugar, la función Caracterizar metodológicamente la propia investigación nos muestra que son los tesistas de Licenciatura y Magíster quienes, en el Desarrollo Teórico, incorporan mayor cantidad de fragmentos que presentan su investigación desde el punto de vista metodológico. En cambio, cuando se trata de describir aspectos metodológicos que el mismo tesista ha propuesto (Describir aspectos metodológicos propuestos), son los tesistas doctorales los más propensos a hacerlo en dicha sección. Creemos que la presencia de estos elementos metodológicos en el Desarrollo Teórico evidencia que, en alguna medida, las Tesis de Lingüística no delimitan claramente los temas de acuerdo con la función que tradicionalmente se ha atribuido a ciertas secciones de un trabajo de graduación. Así, se encontró fragmentos dedicados a la metodología en un apartado, supuestamente, centrado en la revisión de trabajos previos (Paltridge y Starfield, 2007; Rüger, 2011).

Además, esta agrupación de Tesis de Licenciatura y Magíster frente a la de Doctorado en cuanto a aspectos metodológicos se podría explicar si se consideran las exigencias propias de cada grado académico. Así, tanto en Licenciatura como en Magíster no sería necesaria la construcción de aspectos metodológicos por parte del autor, sino solo su descripción, pues se trata de trabajos con menor grado de aportación de conocimientos específicos (UDLAP, 2012); mientras que dicho requerimiento sería fundamental en el caso de una tesis doctoral.

En esta misma línea, las funciones Indicar objetivos y Plantear hipótesis o supuestos, que resultan de frecuencia media en Licenciatura y Magíster, y baja en Doctorado, reafirman el hecho de que en las Tesis de Doctorado se presentan menor cantidad de descripciones metodológicas en la sección Desarrollo Teórico. Así, los tesistas del nivel superior se apegan más a lo que idealmente se debe abordar en la sección dedicada al sustento teórico de la Tesis, esto es, dar cuenta de las nociones teóricas más relevantes para la investigación (UDLAP, 2012). Asimismo, Indicar objetivos es una función que aparece en el marco de algunos análisis de género, en términos de pasos y movidas, que se han hecho de la tesis doctoral (Kwan, 2006). En este sentido Kwan (2006) reconoce que la Estrategia 3A (Anunciando el objetivo de la investigación) de la Movida 3 (Anunciando la propia investigación) es opcional, lo que es coincidente con la baja frecuencia que presenta la función Indicar objetivos en las Tesis de Doctorado de nuestro corpus.

Otra función interesante de comentar es Indicar aportes, que muestra que el tesista de Doctorado aprovecha mayormente el espacio de Desarrollo Teórico para demostrar que puede hacer aportes a su disciplina, posicionándose como un "reasonable, intelligent, co-player in the community's efforts to construct knowledge" (Hyland, 2004:13). Esto permite comprobar que, a pesar de que el Desarrollo Teórico se ha caracterizado tradicionalmente como un apartado en el que lo prin- 
cipal son los aportes de otros al tema tratado, igualmente el tesista posiciona sus propias contribuciones. En consecuencia, a partir de esta investigación empírica se puede afirmar que es necesario matizar ciertas afirmaciones que provienen de investigaciones prescriptivas.

Por último, la frecuencia de aparición de la función Realizar recomendaciones demuestra que son los tesistas doctorales los más propensos a persuadir al lector para que realice alguna acción (frecuencia alta en Doctorado y media en Licenciatura y Magíster). Esta mayor cantidad de directivos, en términos de Hyland (2005), muestra una mayor preocupación por guiar al lector mediante recomendaciones tales como "Ver tabla 29" [PUCVDOCT2005_12].

Ahora, a modo de síntesis, en la Tabla III se sistematizan los resultados de las funciones de la Fuente Propia en la sección Desarrollo Teórico mediante la construcción de patrones de uso basados en la frecuencia de aparición. Esto permite revelar las funciones preferidas en los distintos niveles académicos estudiados:

Tabla III. Funciones de la Fuente Propia más utilizadas en la sección Desarrollo Teórico por nivel académico.

\begin{tabular}{|l|l|l|}
\hline \multicolumn{1}{|c|}{ LICENCIATURA } & \multicolumn{1}{c|}{ MAGÍSTER } & \multicolumn{1}{c|}{ DOCTORADO } \\
\hline $\begin{array}{l}\text { Indicar organización> } \\
\text { Justificar > Emitir juicios }\end{array}$ & $\begin{array}{l}\text { Indicar organización }> \\
\text { Justificar > Problematizar }\end{array}$ & $\begin{array}{l}\text { Indicar organización }> \\
\text { Justificar > Explicar un } \\
\text { artefacto icónico propio }\end{array}$ \\
\hline
\end{tabular}

A partir de la Tabla III es posible advertir que las dos primeras preferencias son las mismas en los tres niveles académicos, existiendo una diferencia en la tercera función. Por una parte, esto indica que no existen patrones de uso constantes, sino que cada nivel presenta un patrón de uso diferente. Ello da cuenta de que, en la sección Desarrollo Teórico, al atribuir la información a una Fuente Propia, existe un modo particular de comunicar el conocimiento en cada nivel. Por otra parte, el establecimiento de patrones indica que, en la sección Desarrollo Teórico, en todos los grados académicos, el tesista se muestra como el responsable de la información, principalmente para organizar su trabajo, vale decir, en un rol de arquitecto (Tang y John, 1999); y para realizar justificaciones de lo que hace o deja de hacer en su investigación. Ejemplos de estas funciones de la Fuente Propia más utilizadas son los siguientes:

\section{a) Indicar organización:}

"A continuación, mencionaremos las técnicas de investigación más destacadas y utilizadas en nuestro campo de trabajo" [UDECMAG2007_38]. 


\section{b) Justificar:}

"En la prueba de comprensión que diseñamos sólo incluimos el primer grupo de inferencias, por una parte porque corresponden al nivel de comprensión que nos interesa y, por otra parte, por ser más fáciles de elicitar y evaluar" [PUCVMAG2007_17].

\section{c) Emitir juicios:}

"Opino que la palabra acrónimo nos es muy útil, pero no para dar nombre a lo que ya lo tiene (al menos en español), como las siglas (sean pronunciables o no, deletreables o no), sino para abarcar las palabras que se forman de manera arbitraria con partes de otras palabras, sean iniciales o no, pero con la condición de que al menos algunas de las letras no sean iniciales (puesto que, si lo fueran, se llamarían siglas)" [UDECMAG2006_29].

\section{d) Problematizar:}

"Ahora bien ¿qué sucede cuando aparece un verbo concreto con un participante inanimado? tal como en el siguiente caso: a) La piedra voló por los aires" [PUCVDOCT2004_9].

\section{e) Explicar un artefacto icónico propio:}

"Las tablas presentadas anteriormente corresponden a estudios realizados en España, por esta razón incluyen segmentos que no son fonemas en el dialecto español de Chile como es el caso de la interdental /T/ y de la alveolar /z/" [UDECMAG2007_45]

Para finalizar la presentación de nuestros hallazgos, y a modo de síntesis, en la Figura 1 se muestra más claramente cómo se comunica el conocimiento en la sección Desarrollo Teórico de las Tesis de cada nivel, a partir del uso de las funciones de la Fuente Propia. A diferencia de la información presentada en la Tabla III, en la Figura 1 no se muestran preferencias de uso, sino que se da cuenta de todas las funciones que se usan en cada grado académico, en la sección en cuestión. Dicho de otro modo, se presenta cuáles son las funciones posibles con las que el tesista de distintos grados académicos se atribuye la información en el apartado Desarrollo Teórico. 


\begin{tabular}{|c|c|c|}
\hline LICENCIATURA & MAGÍSTER & DOCTORADO \\
\hline $\begin{array}{l}\text { Caracterizar metodológica- } \\
\text { mente la propia investigación }\end{array}$ & $\begin{array}{l}\text { Definir o explicar un concep- } \\
\text { to o teoría. }\end{array}$ & $\begin{array}{l}\text { Definir o explicar un concep- } \\
\text { to o teoría. }\end{array}$ \\
\hline $\begin{array}{l}\text { Describir aspectos metodoló- } \\
\text { gicos propuestos }\end{array}$ & $\begin{array}{l}\text { Caracterizar metodológica- } \\
\text { mente la propia investigación }\end{array}$ & $\begin{array}{l}\text { Caracterizar metodológica- } \\
\text { mente la propia investigación }\end{array}$ \\
\hline $\begin{array}{l}\text { Ejemplificar los resultados o } \\
\text { hallazgos de la investigación }\end{array}$ & $\begin{array}{l}\text { Describir aspectos metodoló- } \\
\text { gicos propuestos }\end{array}$ & $\begin{array}{l}\text { Describir aspectos metodoló- } \\
\text { gicos propuestos }\end{array}$ \\
\hline $\begin{array}{l}\text { Emitir juicios sobre algún as- } \\
\text { pecto de la Tesis }\end{array}$ & $\begin{array}{l}\text { Emitir juicios sobre algún as- } \\
\text { pecto de la Tesis }\end{array}$ & $\begin{array}{l}\text { Ejemplificar resultados o ha- } \\
\text { llazgos }\end{array}$ \\
\hline $\begin{array}{l}\text { Enmarcar la investigación o } \\
\text { un concepto relevante para } \\
\text { ella }\end{array}$ & $\begin{array}{l}\text { Enmarcar la investigación o } \\
\text { un concepto relevante para } \\
\text { ella }\end{array}$ & $\begin{array}{l}\text { Emitir juicios de algún aspec- } \\
\text { to de la Tesis } \\
\text { Explicar un artefacto icónico }\end{array}$ \\
\hline $\begin{array}{l}\text { Explicar un artefacto icónico } \\
\text { propio }\end{array}$ & $\begin{array}{l}\text { Explicar un artefacto icónico } \\
\text { propio }\end{array}$ & $\begin{array}{l}\text { propio } \\
\text { Exponer resultados o hallaz- }\end{array}$ \\
\hline Exponer resultados o hallaz- & Indicar aportes & gos de la investigación \\
\hline gos de la investigación & Indicar dificultades & Indicar aportes \\
\hline Indicar aportes & Indicar limitaciones & Indicar dificultades \\
\hline Indicar dificultades & Indicar objetivos & Indicar limitaciones \\
\hline Indicar objetivos & Indicar organización & Indicar notación \\
\hline Indicar organización & Indicar procedimientos & Indicar objetivos \\
\hline Indicar vacíos/ necesidades en & Indicar vacíos/ necesidades en & Indicar organización \\
\hline Justificar & el conocimiento & Indicar procedimientos \\
\hline Plantear hipótesis o supuestos & fustm & Indicar proyecciones \\
\hline Problematizar & Problematizar & $\begin{array}{l}\text { Indicar vacíos en el conoci- } \\
\text { miento }\end{array}$ \\
\hline Proponer aspectos teóricos & Realizar interpretaciones $\mathrm{o}$ & Introducir un concepto \\
\hline Realizar interpretaciones o & deducciones & Justificar \\
\hline Relizar recton & Realizar recomendaciones & Plantear hipótesis o supuestos \\
\hline Sintetizar algún aspecto de la & $\begin{array}{l}\text { Sintetizar algún aspecto de la } \\
\text { Tesis }\end{array}$ & $\begin{array}{l}\text { Presentar desarrollo del aná- } \\
\text { lisis }\end{array}$ \\
\hline & & Problematizar \\
\hline & & Proponer aspectos teóricos \\
\hline & & $\begin{array}{l}\text { Realizar interpretaciones o } \\
\text { deducciones }\end{array}$ \\
\hline & & Realizar recomendaciones \\
\hline & & $\begin{array}{l}\text { Sintetizar algún aspecto de la } \\
\text { Tesis }\end{array}$ \\
\hline & & Superar dificultad \\
\hline
\end{tabular}

Figura 1. Funciones de la Fuente Propia utilizadas para comunicar el conocimiento en la sección Desarrollo Teórico. 


\section{CONCLUSIONES}

Como se ha señalado previamente, una de las características centrales del género Tesis es que mediante él se construye (Badley, 2009) y comunica el conocimiento (Arnoux, 2006; Meza, 2013, 2015). Si bien este aspecto es fundamental para la inclusión del estudiante en la comunidad discursiva, son muy pocos los trabajos que indagan, a partir de un estudio de corpus, en la comunicación del conocimiento propio en el género Tesis, y en las diferencias entre los grados de Licenciatura, Magíster y Doctorado en este género.

En este contexto, creemos que la presente propuesta de investigación y el análisis realizado resultan de suma utilidad. Esto porque, primero, permitió capturar la organización comunicativa de una sección fundamental de la Tesis, estableciendo similitudes y diferencias entre distintos grados académicos del género Tesis de Lingüística. De esta manera, se pudo evidenciar las funciones específicas con las que el autor de la Tesis incorpora su propia voz en la sección Desarrollo Teórico. Además, el estudio expuesto permite ampliar y matizar la comprensión de una sección tan relevante de la Tesis como es el Desarrollo Teórico. Si bien tradicionalmente esta sección se ha comprendido como la presentación de investigaciones realizadas previamente por otros autores (Phillips y Pugh, 2005; Paltridge y Starfield, 2007; Rüger, 2011), en este trabajo se demuestra empíricamente y a nivel granular que en dicha sección se muestran aportes del propio autor, presentando, incluso, planteamientos teóricos propios.

A fin de hacer más evidentes los aportes de este trabajo, a continuación, sobre la base de los resultados obtenidos, se responde cada una de las preguntas de investigación que se plantearon inicialmente. Así, en primer lugar, con respecto a la interrogante sobre las funciones con las cuales se comunica el conocimiento propio en la sección Desarrollo Teórico de las Tesis del corpus TeLing3N, es posible señalar que se utilizan 27 de las 31 funciones del conocimiento propio, lo que equivale al $87,1 \%$.

En segundo lugar, para responder a la pregunta que indagaba sobre la variación entre grados académicos en las funciones utilizadas para comunicar el conocimiento propio en la sección Desarrollo Teórico, es posible señalar que Licenciatura y Magíster presentan 19 de las 27 funciones encontradas; mientras que en Doctorado se utilizan 26 de dicho total. Entonces, al comparar entre los tres grados, el más alto es el único que tiene funciones exclusivas. Ello se podría explicar a partir del mayor grado de conocimiento, inserción y consolidación del estudiante de doctorado, lo que tiene como consecuencia un mayor dominio de los recursos lingüísticos necesarios para comunicar el conocimiento propio en un género específico y en una sección determinada.

La frecuencia de aparición de las distintas funciones de la Fuente Propia también permite realizar una serie de distinciones entre grados académicos, a saber: 
entre Licenciatura y Magíster versus Doctorado, entre pre y postgrado y entre los tres grados académicos. Por una parte, a partir del uso de las funciones de la Fuente Propia, se corroboran las diferencias propias del género Tesis en las distintas etapas de una carrera académica; $y$, por otra, confirma que la sección estudiada cumple un propósito comunicativo estable, a pesar de realizarse de diferentes formas, descritas en los resultados, en los distintos grados académicos.

En síntesis, nuestros resultados arrojan evidencia empírica de que en todos los niveles académicos los tesistas se incorporan para atribuirse parte de la información presentada en dicha sección, vale decir, no existen Tesis en las que la sección en cuestión sea construida exclusivamente a partir de lo dicho por otros. Así, la variación entre los distintos niveles está dada por la proporción de la información que el tesista se atribuye a sí mismo y por la variedad de funciones con que lo hace. De ello se desprende que la Tesis no es un género homogéneo, sino que varía de acuerdo con el grado académico en que se desarrolla, por lo que cualquier descripción o aplicación que se haga de este género en su totalidad o de una sección particular debe considerar la variable grado académico.

Sobre la base de estos hallazgos, podemos establecer un vínculo con algunos estudios sobre la autorrepresentación (self-portrayal) del autor en discursos ficcionales y no ficcionales (Cherry, 1988). En este marco, y considerando la idea que sugiere que toda vez que un autor se inserta en su texto crea una persona distinta de la real (Cherry, 1988), se puede afirmar que, en cada una de las Tesis del corpus TeLing3N, el escritor de Tesis crea una persona con algunas características particulares. Por ejemplo, se muestra en su discurso como alguien que es capaz de aportar a su comunidad, que domina los conocimientos de su disciplina, que puede emitir juicios, que es capaz de contradecir investigaciones previas, etc. De este modo, en términos de Cherry (1988), se crea una "máscara teatral", la que, como indican nuestros resultados, sufre algunas modificaciones, dependientes del grado académico en que se desarrolle la Tesis. En este sentido, es posible afirmar que, sobre la base del uso de las funciones de la Fuente Propia, el tesista de Doctorado crearía una máscara de experto, conocedor y usuario estratégico de los modos de decir de su disciplina.

En conclusión, la presente investigación se erige como un aporte tanto a la disciplina como a los mismos estudiantes que están realizando su Tesis. En el primer caso, aporta con valiosa información empírica sobre un género fundamental en la formación a nivel mundial en tres niveles académicos; además, se explora una sección de la Tesis escasamente abordada (Silva, 2012). En el segundo caso, si bien existen manuales que asisten en la escritura académica, esta investigación contribuye a mejorar la caracterización a partir de datos empíricos, lo que se configura como un aporte para abordar la escritura de la sección Desarrollo Teórico.

Tenemos la certeza de que todos estos hallazgos constituyen un material valiosísimo para ayudar a estudiantes de distintos grados académicos en su proceso 
de escritura del Desarrollo Teórico de su Tesis, ya que, a nuestro juicio, conocer la utilización estratégica de las funciones del discurso, asociadas a una fuente de la información específica, resulta de gran valor para quienes deben escribir su trabajo final de grado, pues este modo de abordar la escritura de una Tesis le indica claramente a un estudiante cómo dar cuenta de sus contribuciones. Más específicamente, le señala al tesista con qué funciones exactamente puede atribuirse la información a sí mismo, según el grado académico en que realiza su trabajo.

\section{REFERENCIAS}

Abasi, A. \& Graves, B. (2008). Academic literacy and plagiarism: Conversations with international graduate students and disciplinary professors. Journal of English for Academic Purposes, 7, 221-233.

Albert, María José. (2006). La investigación educativa. Claves teóricas. Madrid, España: McGraw-Hill.

Aleskerov, Fuad. (2009). How to prepare and write PhD and MS thesis. Automation and Remote Control, 70, 1928-1938.

Arnoux, Elvira. (2006). Incidencia de la lectura de pares y expertos en la reescritura de tramos del trabajo de Tesis. Revista de Lingüistica Teórica y Aplicada, 44, 95-118.

Arnoux, Elvira; Borsinger, Ann; Carlino, Paula; Di Stefano, Mariana; Pereira, Cecilia y Silvestre, Adriana. (2005). La intervención pedagógica en el proceso de escritura de Tesis de posgrado. Disponible en: http://www.escrituraylectura.com. ar/posgrado/articulos.htm

Badley, Graham. (2009). Academic writing as shaping and re-shaping. Teaching in Higher Education, 14, 209-219.

Blessing, Lucienne y Chakrabarti, Amaresh. (2009). DRM, a Design Research Methodology. Dordrecht, Holland: Springer.

Bui, Yvonne. (2013). How to Write a Master's Thesis. Los Angeles, Estados Unidos: Sage.

Bunton, David (2002). Generic moves in PhD thesis introductions. En J. Flowerdew (Ed.), Academic discourse (pp. 57-75). Harlow, England: Pearson.

Carlino, Paula. (2005) ¿Por qué no se completan las Tesis en los posgrados? Obstáculos percibidos por maestrandos en curso magistri exitosos. Educere, 9, 415420.

Cherry, Roger. (1988). Ethos vs. persona: self-representation in written discourse. Written Communication, 5, 251-276.

Colás, Pilar; Buendía, Leonor y Hernández, Fuensanta. (2009). Competencias cientificas para la realización de una Tesis doctoral. Barcelona, España: Da Vinci.

Dardy, Claudine; Ducard, Dominique y Maingueneau, Dominique. (2002). Un 
genre universitaire. Le rapport de soutenance de thèse. Lille, Francia: Septentrion. Hanania, Edith y Akhtar, Karima. (1985). Verb Form and Rhetorical Function in Science Writing: A Study of MS Theses in Biology Chemistry and Physics. The ESP Journal, 4, 49-58.

Hidalgo, Cecilia y Passarella, Virginia. (2009). Encuesta "Escritura y producción de conocimiento en carreras de Posgrado" administrada a estudiantes de posgrado de diversas instituciones de Argentina y Chile (2005-2006). En E. Arnoux (Ed.), Escritura y producción de conocimiento en las carreras de posgrado (62-73). Buenos Aires, Argentina: Santiago Arcos Editor.

Hyland, Ken. (2003). Second Language Writing. Cambridge: Cambridge University Press.

Hyland, Ken. (2004). Disciplinary discourses. Michigan, Estados Unidos: The University of Michigan Press.

Hyland, Ken. (2005). Stance and engagement: a model of interaction in academic discourse. Discourse Studies, 7, 173-192.

Koutsantoni, Dimitra. (2006). Rhetorical strategies in engineering research articles and research theses: Advanced academic literacy and relations of power. Journal of English for Academic Purposes, 5, 19-36.

Kwan, Becky. (2006). A genre analysis of literature reviews in doctoral theses. Unpublished English Communication Doctoral Thesis. Hong Kong, China: University of Hong Kong.

Landis, J. Richard y Koch, Gary. (1977). The measurement of observer agreement for categorical data. Biometrics, 33, 159-174.

Lovitts, Barbara y Wert, Ellen. (2009). Developing quality dissertations. A graduate student's guide to achieving excellence. Sterling, Estados Unidos: Stylus Publishing.

Meza, Paulina. (2013). La comunicación del conocimiento en las secciones de Tesis de lingüística: determinación de la variación entre grados académicos. Tesis doctoral de Lingüística. Valparaíso, Chile: Pontificia Universidad Católica de Valparaíso.

Meza, Paulina. (2015). La comunicación del conocimiento en el género Tesis de Lingüística: Comparación entre grados académicos. En G. Parodi y G. Burdiles (Eds.), Leer y escribir en contextos académicos y profesionales (pp. 67-112). Santiago, Chile: Planeta.

Moyano, Estela. (2000). Comunicar ciencia. Buenos Aires, Argentina: Secretaría de Investigaciones. Universidad Nacional de Lomas de Zamora.

Paltridge, Brian y Starfield, Sue. (2007). Thesis and dissertation writing in a second language: A handbook for supervisors. Londres, England: Routledge.

Pereira, Cecilia y Di Stefano, Mariana. (2007). El taller de escritura en posgrado: Representaciones sociales e interacción entre pares. Revista Signos. Estudios de Lingüística, 40, 405-430. 
Petrić, Bojana. (2007). Rhetorical functions of citations in high and low-rated master's thesis. Journal of English for Academic Purposes, 6, 238-253.

Phillips, Estelle y Pugh, Derek (2005). How to get a PhD. A handbook for students and their supervisor. Maidenhead, England: Open University Press.

Rüger, Stefan. (2011). How to write a good PhD thesis and survive the viva. Disponible en http://people.kmi.open.ac.uk/stefan/thesis-writing.pdf

Salinas, Dagoberto. (2010). ¿Cuántos y a quiénes preguntar? Una aproximación al muestreo cuantitativo y cualitativo en investigación social y educacional. Valparaíso, Chile: Ediciones Universitarias de Valparaíso.

Savio, Ana. (2010). Las huellas del autor en el discurso académico: un estudio sobre Tesis de psicoanalistas argentinos. Lenguaje, 38, 563-590.

Sierra, Restituto. (2003). Tesis doctorales y trabajos de investigación cientifica: metodología general de su elaboración y documentación. Madrid, España: Paraninfo.

Silva, Muriel. (2012). Caracterización del apartado Marco Teórico a partir de movidas y estrategias en Tesis de Magister de Lingüistica y Filosofía. Tesis de Licenciatura en Lingüística. Valparaíso, Chile. Pontificia Universidad Católica de Valparaíso.

Tang, Ramona y John, Suganthi. (1999). The 'I' in identity: Exploring writer identity in student academic writing through the first person pronoun. English for SpecificPurposes, 18, 23-39.

Tapia, Mónica y Marinkovich, Juana. (2011). Representaciones sociales sobre la escritura de la tesis en la formación académica inicial en el área de las Ciencias del Mar. Onomázein, 24, 273-297.

Tapia, Mónica y Burdiles, Gina. (2012). La organización retórica del marco referencial en Tesis de Trabajo Social. Alpha (Osorno), 35, 169-184.

Thompson, Paul. (2005). Points of focus and position: Intertextual reference in PhD theses. Journal of English for Academic Purposes, 4, 307-323.

Turabian, Kate. (2007). A manual for writers of research papers, theses, and dissertations: Chicago style for students and researchers. Chicago, Estados Unidos: The University of Chicago Press.

UDLAP (2012). Tesina. Disponible en :http://printfu.org/read/tesina-7f38. html?f=1 qeXpurpn6Wih-SUpOGunK-PudHi39PViqDjrJqolsyXqNimp6efltDZ5OKjnaPd7OST4dPixuSW0uih0tzo2NbbyuDG28ejy8re5tvd2Mva4Mje2Ora5smU1tvV0-V49zZzeLZ1OHY0dXmyuGj2trgztrQpNXYzoer $7 \mathrm{w}$

USACH (2008). Manual para normalización de trabajos de titulación o Tesis de grado de la Biblioteca Central de la Universidad de Santiago de Chile. Disponible en: https://biblioteca.usach.cl/arch/doc/reg_Tesis/introduccion.pdf

Venegas, René; Meza, Paulina y Martínez, Juan. (2013). Procedimientos discursivos en la atribución del conocimiento en tesis de lingüística y filosofía en dos niveles académicos. Revista de Lingüística Teórica y Aplicada, 51, 153-179. 
Venegas, René; Núñez, María; Zamora, Sofía; Santana, Andrea. (2015). Escribir desde la pedagogía del género. Guías para el Trabajo Final de Grado de Licenciatura. Valparaíso, Chile: Ediciones Universitarias de Valparaíso.

Yasmin, Tayyaba y Mahmood, Muhammad. (2017). A Comparative Analysis of Writer's Identity in Pakistani Research Theses of English and Psychology. International Journal of English Linguistics, 7, 255-260. 